\title{
The BEN domain is a novel sequence- specific DNA-binding domain conserved in neural transcriptional repressors
}

\author{
Qi Dai, ${ }^{1,3}$ Aiming Ren, ${ }^{2,3}$ Jakub O. Westholm, ${ }^{1}$ Artem A. Serganov, ${ }^{2}$ Dinshaw J. Patel, ${ }^{2}$ \\ and Eric C. Lai ${ }^{1,4}$ \\ ${ }^{1}$ Department of Developmental Biology, ${ }^{2}$ Department of Structural Biology, Sloan-Kettering Institute, New York, \\ New York 10065, USA
}

\begin{abstract}
We recently reported that Drosophila Insensitive (Insv) promotes sensory organ development and has activity as a nuclear corepressor for the Notch transcription factor Suppressor of Hairless [ $\mathrm{Su}(\mathrm{H})]$. Insv lacks domains of known biochemical function but contains a single BEN domain (i.e., a "BEN-solo" protein). Our chromatin immunoprecipitation (ChIP) sequencing (ChIP-seq) analysis confirmed binding of Insensitive to $\mathrm{Su}(\mathrm{H})$ target genes in the Enhancer of split gene complex [E(spl)-C]; however, de novo motif analysis revealed a novel site strongly enriched in Insv peaks (TCYAATHRGAA). We validate binding of endogenous Insv to genomic regions bearing such sites, whose associated genes are enriched for neural functions and are functionally repressed by Insv. Unexpectedly, we found that the Insv BEN domain binds specifically to this sequence motif and that Insv directly regulates transcription via this motif. We determined the crystal structure of the BEN-DNA target complex, revealing homodimeric binding of the BEN domain and extensive nucleotide contacts via $\alpha$ helices and a C-terminal loop. Point mutations in key DNA-contacting residues severely impair DNA binding in vitro and capacity for transcriptional regulation in vivo. We further demonstrate DNA-binding and repression activities by the mammalian neural BEN-solo protein BEND5. Altogether, we define novel DNA-binding activity in a conserved family of transcriptional repressors, opening a molecular window on this extensive gene family.
\end{abstract}

[Keywords: BEN domain; neurogenesis; transcriptional repression]

Supplemental material is available for this article.

Received January 3, 2013; revised version accepted February 6, 2013.

Sequence-specific transcription factors coordinate and drive all programs of development and physiology, and a few dozen families of sequence-specific DNA-binding folds have been characterized to date (Yusuf et al. 2012). With the passing years, the recognition of novel DNAbinding domains has become increasingly infrequent. In particular, the latest DNA-binding domains were recognized from bacterial or fungal species (Boch et al. 2009; Moscou and Bogdanove 2009; Wiedenheft et al. 2009; Lohse et al. 2010), with few new domains described in metazoans. Nevertheless, the observation that many uncharacterized sequence motifs emerge from multigenome alignments and genome-wide data collection efforts (Stark et al. 2007; Lindblad-Toh et al. 2011; Neph et al. 2012) indicates that many binding sites remain to be connected to their cognate transcription factors. Undoubtedly, many of these correspond to sites for the hundreds of

\footnotetext{
${ }^{3}$ These authors contributed equally to this work.

${ }^{4}$ Corresponding author

E-mail laie@mskcc.org

Article published online ahead of print. Article and publication date are online at http://www.genesdev.org/cgi/doi/10.1101/gad.213314.113.
}

annotated DNA-binding proteins that have yet to be characterized, but perhaps some of them may represent target sites of proteins whose DNA-binding activity is not evident from a primary sequence.

The Drosophila peripheral nervous system has been an exemplary model system for studying the sequential adoption of cell fates during development (Lai and Orgogozo 2004). The capacity for neurogenesis within the epidermis is conferred by the spatially patterned expression and activity of proneural basic helix-loop-helix (bHLH) activator proteins in proneural clusters. For example, the bHLH factors Achaete and Scute are responsible for initiating the development of the several hundred external mechanosensory organs that decorate the body surface. Local cell interactions mediated by the Notch signaling pathway subsequently restrict neural potential to individual sensory organ precursors (SOPs), which then execute a stereotyped lineage to generate the cells of a mature sensory organ comprised of a neuron and several nonneural accessory cells (Lai 2004). Each cell division in the neural lineages is asymmetric and driven by Notch signaling such that loss-of-function and gain-of-function 
of the Notch pathway can induce reciprocal, symmetric cell fate outcomes. In particular, the neuron is the ultimate product of multiple precursor cells that successfully avoid activating Notch signaling.

A new component in Drosophila neurogenesis emerged from the recovery of Insensitive (Insv) in a molecular screen for genes specifically expressed in proneural clusters (Reeves and Posakony 2005). The only recognizable domain on Insv is a BEN domain. Named after instances in the BANP, E5R, and NAC1 proteins, this bioinformatically recognized motif is widely distributed among metazoans and viruses (Abhiman et al. 2008). Some BEN proteins contain other motifs linked to chromatin functions, but Insv is the prototype of the "BEN-solo" family that lacks other recognizable protein domains. We recently showed that Insv is a nuclear factor that promotes peripheral neurogenesis and inhibits Notch signaling (Duan et al. 2011). In particular, Insv has activity as a direct corepressor for the Notch pathway transcription factor Suppressor of Hairless [Su(H)] and localizes in vivo to a number of Notch target genes located in the Enhancer of split-Complex [E(spl)-C] (Duan et al. 2011).

In this study, we examined the genomewide occupancy of Insv during two stages of Drosophila embryogenesis. We confirmed binding of Insv to Notch target genes in the $E(s p l)-C$; however, Insv bound thousands of regions that did not contain consensus $\mathrm{Su}(\mathrm{H})$-binding sites. Instead, these regions defined a novel sequence motif that was enriched among genes involved in neurogenesis, and genes with Insv occupied-motifs were broadly up-regulated in insv mutants. Surprisingly, we found that Insv bound to this motif directly via the BEN domain, and we went on to solve the structure of the BEN domain in complex with its DNA target. Extensive mutational analysis confirmed the structural details of BEN domain interactions with the DNA backbone and with critical base-specific contacts that explain the affinity of Insv for its cognate DNA target. Although BEN domains are only loosely related to each other, knowledge of the Insv structure allowed us to recognize other BEN proteins that share its base-specific amino acids. In particular, we show that mammalian BEND5 is also a sequence-specific transcriptional repressor that regulates neurogenesis, despite very limited overall homology with Insv. In summary, our work defines a novel conserved family of metazoan transcription factors and provides direction to the study of the other $>100$ annotated BEN domain proteins (Abhiman et al. 2008).

\section{Results}

Genome-wide analysis reveals a novel DNA motif associated with transcriptional repression by Insv

We recently reported that the BEN-solo factor Insensitive (Insv) is a corepressor for the Notch pathway transcription factor Suppressor of Hairless $[\mathrm{Su}(\mathrm{H})]$ and opposes Notch function during peripheral nervous system development (Duan et al. 2011). Evidence for this included protein-protein interaction between Insv and $\mathrm{Su}(\mathrm{H})$ and chromatin immunoprecipitation (ChIP) of endogenous
Insv to multiple Notch/Su(H)-regulated targets resident in the $E(s p l)$-C, including both bHLH repressor and Bearded family genes (Duan et al. 2011). Insv mutants exhibit mild sensory organ loss and shaft-to-socket transformations within the sensory lineage, phenotypes that are exacerbated by elevation of haploinsufficiency of the characterized $\mathrm{Su}(\mathrm{H})$ corepressor Hairless.

To gain insight into regulatory targets of Insv genomewide, we performed Insv ChIP sequencing (ChIP-seq) using 2.5- to 6.5-h and 6.5- to 12 -h embryos. These data revealed 5364 and 2390 peaks, respectively, relative to control IgG ChIP-seq (Supplemental Table 1). The specificity of Insv binding was well-illustrated at the $E(s p 1)$-C, where Insv peaks were concentrated across the upstream regions of most of these Notch-regulated genes (Fig. 1A), whereas binding of Insv to the flanking hundreds of kilobases was sparse. Despite this, neither high-affinity (YGTGRGA) nor low-affinity (RTGRGAR) consensus sequences for $\mathrm{Su}(\mathrm{H})$ (Nellesen et al. 1999) were enriched among Insv peaks genome-wide, and the majority of regions strongly bound by Insv lacked $\mathrm{Su}(\mathrm{H})$-binding sites (Supplemental Fig. 1).

An example of such a region is shown in Figure 1B, where both Insv ChIP-seq data sets reported on strong binding to a conserved genomic region located close to the promoter of the Notch modulator fringe. To understand the basis of chromatin association of Insv in the absence of $\mathrm{Su}(\mathrm{H})$ sites, we performed de novo motif analysis using Weeder (Pavesi et al. 2001) and MEME (Bailey and Elkan 1994), querying Insv peaks of various classes. We considered all bound regions, those regions that were exclusive of $\mathrm{Su}(\mathrm{H})$-binding sites, and regions exclusive of highly occupied target (HOT) regions (Roy et al. 2010). Analysis by MEME reported a novel semipalindromic motif (TCYAATHRGAA) as the most highly enriched sequence (Fig. 1C), while Weeder reported the perfect palindromic sequence (CCAATTGG). Indeed, these motifs were enriched even without having to preclude peaks containing $\mathrm{Su}(\mathrm{H})$ sites. Genome-wide, the Insv ChIP peaks bearing the TCYAATHRGAA motif were preferentially located in proximity to transcriptional start sites in both data sets (Fig. 1D; Supplemental Fig. 2). In addition, this motif was preferentially enriched among more strongly bound sites in both ChIP-seq data sets, supporting its biological relevance (Fig. 1E; Supplemental Fig. 1).

We confirmed in vivo binding to these genomic regions by endogenous Insv. We selected Insv ChIP-seq peaks from repo, vestigial, mir-263a, fringe, grainyhead, hamlet, tramtrack, Syt1, Dlic2, Chinmo, and fne (found in neurons) for verification by ChIP-qPCR. All of these are genes involved in nervous system development, and none of their Insv peaks contained even low-affinity $\mathrm{Su}(\mathrm{H})$ sites. Compared with a control region from CG34245, all of these showed strong Insv binding, with most in excess of 50-fold enrichment above ChIP with control IgG (Fig. 1F). We confirmed this to be due to Insv, since parallel ChIPqPCR assays from insv-null embryos showed no binding to any of these Insv target regions (Fig. 1F).

We cross-referenced the genes whose Insv ChIP peaks included the identified motif with the FlyAtlas database 

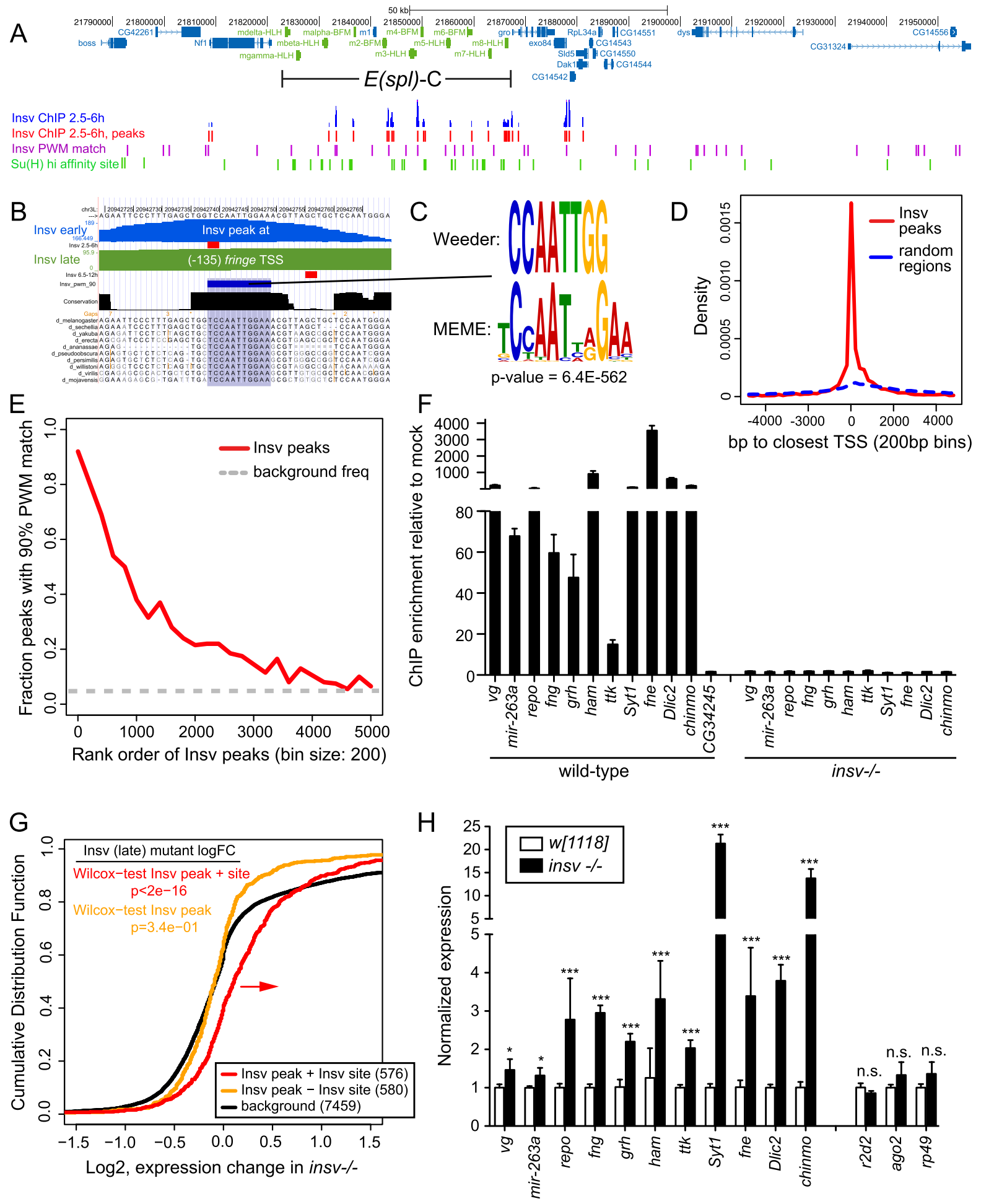

Figure 1. ChIP-seq revealed a novel motif associated with transcriptional repression by Drosophila Insv. $(A)$ Insv exhibits extensive binding to $\mathrm{N} / \mathrm{Su}(\mathrm{H})$-regulated enhancers throughout the $E(s p l)-\mathrm{C}$ (green highlighted genes). (B) Insv binding to the fringe promoter. University of California at Santa Cruz (UCSC) Genome Browser view shows raw ChIP-seq signals from 2.5- to 6.5-h ("early," blue track) and 6.5- to 12-h ("late," green track) embryos; the processed peak locations are marked with red boxes. These peaks coincide with a well-conserved palindromic motif. $(C)$ De novo motif analysis from both early and late data sets defines related motifs that are highly enriched among Insv-bound regions. $(D)$ Insv peaks that bear this motif are strongly enriched near annotated transcription start sites (red line). The dashed blue line shows distances from 100,000 random genomic regions to the closest transcription starts. This analysis is from the early time point, but the same is true for the later time point (Supplemental Fig. 2) ( $\chi^{2} P$-value $\left.<2.2 \times 10^{-16}\right)$. (E) The Insv motif is preferentially localized in stronger Insv peaks in both early (here) and late embryo (Supplemental Fig. 1) time points. The dashed gray line shows the estimated background frequency of this site; the distributions are significantly different. $(F)$ ChIP-qPCR validates binding of Insv to target genes bearing this motif; absence of signals in insv ${ }^{-1-}$ embryos demonstrates specificity. (G) Cumulative distribution function of gene expression in 6- to 8-h wild-type and insv ${ }^{-/}$embryos. Genes associated with Insv ChIP-seq peaks bearing the identified motif (red line) were generally up-regulated in insv mutants relative to background genes (black line), whereas genes bound by Insv but lacking the motif were not up-regulated (orange line); $P$-values from Wilcoxon rank sum test. $(H)$ qPCR validation of the up-regulation of Insv target genes in insv mutant embryos. $\left(^{\star}\right) P<0.001 ;\left(^{\star \star \star}\right) P<0.0001$. 
of tissue-specific gene expression (Chintapalli et al. 2007) and observed that the strongest enrichments of Insv peaks occurred near neural-expressed genes; namely, brain $\left(7.1 \times 10^{-5}\right)$ and thoracicoabdominal ganglion $\left(5.6 \times 10^{-4}\right)$ (Supplemental Table 2). Gene ontology (GO) analysis of genes in proximity to Insv peaks revealed that the most highly enriched categories included several biological functions related to neural development (e.g., nervous system development: $1.9 \times 10^{-27}$, neurogenesis: $3.6 \times 10^{-21}$, and generation of neurons: $9.5 \times 10^{-20}$ ) (Supplemental Table 3). We also note that among molecular function GO categories, Insv peaks were strongly enriched in transcription factors $\left(5.9 \times 10^{-18}\right)$. Altogether, these analyses indicated at the genome-wide level that Insv is broadly bound to genes that are expressed in the nervous system and/or function in neural development, consistent with our knowledge of Insv as a regulator of neural development (Duan et al. 2011).

We assessed the functional consequence of these sites by performing microarray analysis on wild-type and insv mutant embryos. We observed that genes associated with Insv peaks containing TCYAATHRGAA motifs were preferentially up-regulated in the absence of Insv (Fig. 1G). In contrast, Insv peaks that lacked TCYAATHRGAA motifs were not generally up-regulated, implying a specific functional consequence of the motif for gene expression. We confirmed these data by quantitative RT-PCR (qRT-PCR) of Insv-bound targets bearing this motif. These tests validated up-regulation of repo, vestigial, mir-263a, fringe, hamlet, grainyhead, tramtrack, chinmo, Dlic2, Syt1, and fne in insv mutant embryos relative to wild type, whereas many control loci were unchanged (Fig. 1H). These effects were consistent with our recent characterization of Insv as a transcriptional corepressor (Duan et al. 2011), although they now suggested a $\mathrm{Su}(\mathrm{H})$-independent mode of recruitment of Insv to chromatin.

Insv is a novel DNA-binding protein that directly mediates transcriptional repression

The core of the restricted consensus identified by Weeder (CCAATTGG) is reminiscent of the CCAAT box involved in general eukaryotic transcriptional activation (Dolfini et al. 2012); however, the Insv-associated site diverges from the extended CCAAT box (GGCCAATCT). We therefore considered whether Insv might associate with this DNA motif directly. Although full-length recombinant Insv was prone to degradation, preparations of GST-Insv tested positively in gel shifts with the enriched sequence motif, providing candidate evidence for unexpected DNAbinding activity of Insv (data not shown).

We subdivided Insv into an $\mathrm{N}$-terminal fragment bearing a putative coiled-coil region (InsvN) and a C-terminal fragment containing the BEN domain (InsvC); both were stable in solution as purified proteins. Gel shifts showed that Insv DNA-binding activity localized to its BEN domain (Fig. 2A). We confirmed the retarded complex contains Insv, as it was supershifted upon addition of Insv antibody (Fig. 2A). We further validated binding of the Insv-BEN domain to additional conserved instances of this motif located within Insv ChIP-seq peaks in repo, mir263a, fringe, grh, ham, and $t t k$ (Fig. 2B). Thus, the Insv BEN domain harbors novel DNA-binding activity.

We sought evidence that Insv is directly recruited to TCYAATHRGAA motifs in vivo. We initially tested this using luciferase reporters under control of the actin promoter, into which we inserted a multimer of Insv-binding sites or point mutant sites that abrogated Insv binding in vitro (Fig. 2C). Coexpression of full-length Insv did not influence the mutant reporter relative to transfection of empty plasmid but repressed the reporter-bearing Insv sites $>10$-fold (Fig. 2D). These tests ruled out the interpretation that Insv might merely be "sticky" as assayed by binding in vitro and demonstrated that these motifs directly confer transcriptional repression via Insv.

Since most Insv-bound genomic regions contain only a single site, we also tested the functional capacity of endogenous Insv-bound target regions. We assayed regions from vg, mir-263a, repo, fringe, grainyhead, hamlet, and tramtrack and found that almost all of them mediated transcriptional repression in response to ectopic Insv (Fig. 2E). Except for the hamlet site, all the other sites reduced by $\sim 2.5$-fold or greater. Therefore, single endogenous Insv-binding sites are functionally relevant.

We wished to use the Insv transcriptional reporter to provide genetic evidence that the Insv BEN domain contacts the Insv-binding site directly. However, expression of the InsvN or InsvC (BEN) domains did not affect the Insv reporter, likely indicating that these fragments no longer had activity as a transcriptional regulator. We consequently fused Insv to the strong viral transactivation domain of VP16 (VP16-AD) in an effort to convert it into a dominant transcriptional activator (Fig. 2F). Strikingly, Insv-VP16 induced several-hundred-fold activation of the Insv-binding site reporter (Fig. 2G). We proceeded to assay VP16 fusions to the InsvN and InsvC domains and observed that the latter yielded strong transcriptional activation of the Insv reporter (Fig. 2G). These results corroborated the gel shift analysis in demonstrating that the BEN domain is a DNA-binding motif.

\section{Crystal structure of the Insv-BEN domain complexed to its DNA target site}

We were keen to obtain structural insight into the unanticipated DNA-binding activity of the Insv BEN domain. We focused on the BEN domain of Insv spanning residues 251-365, for which we were able to purify sufficient quantities of soluble protein. As efforts to crystallize the BEN domain in the free state were unsuccessful, we focused on complex formation with a target duplex containing the consensus palindromic core-binding site. By screening target sequences containing added bases at the $5^{\prime}$ and $3^{\prime}$ ends, we succeeded in growing crystals of the BEN domain complexed with the 13-mer target GTTCCAATTGGAA, which formed a 12-base-pair (bp) self-complementary duplex with 5' G overhangs that diffracted to $1.58 \AA$. The salient X-ray statistics are provided in Table 1.

Two views of the structure of the complex are shown in Figure 3A, with the complex containing two BEN domains 
Dai et al.
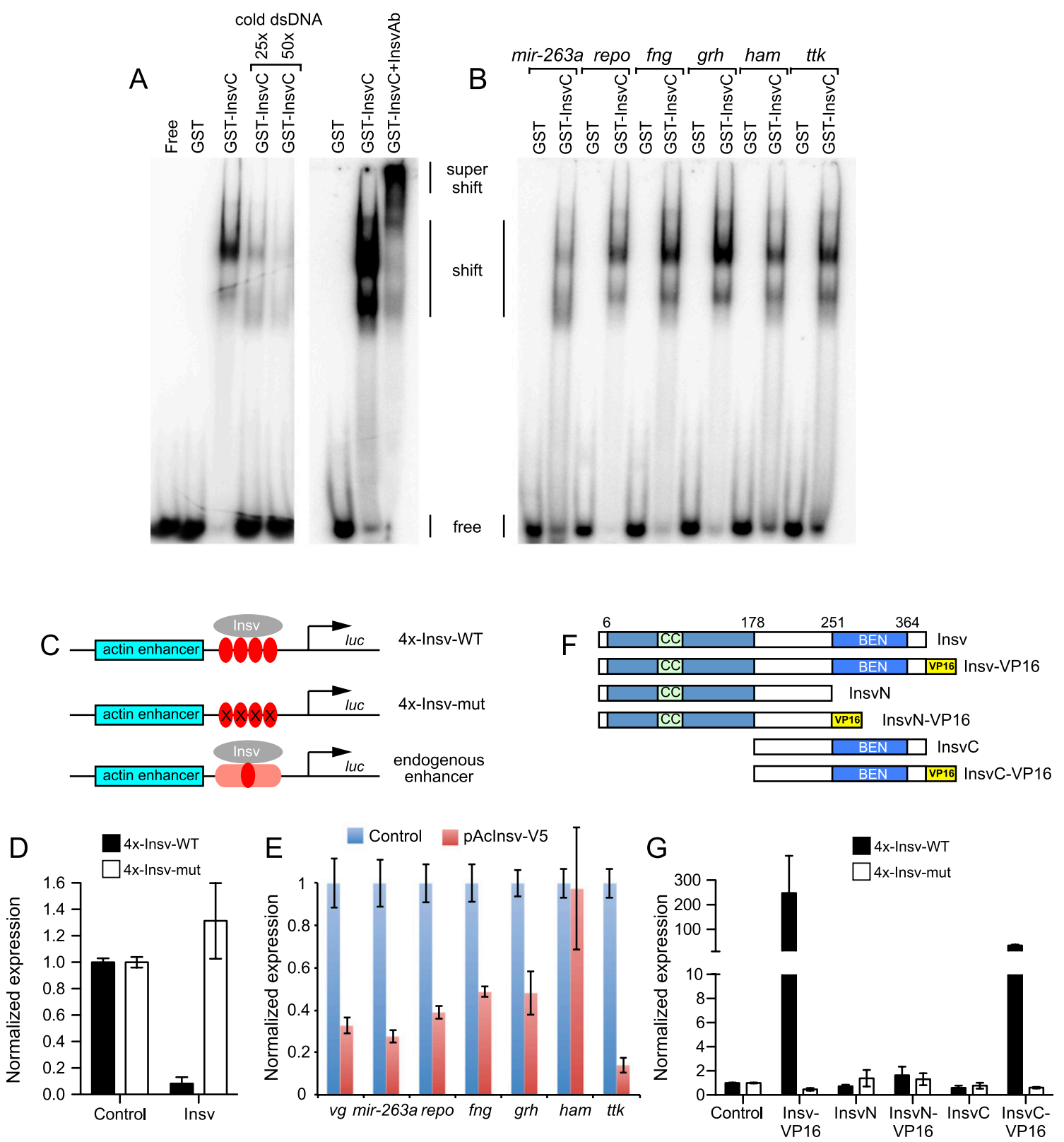

Figure 2. Insv is a novel DNA-binding protein that directly mediates transcriptional repression. $(A)$ Gel shift analysis with radiolabeled probes demonstrates specific binding of the Insv BEN domain to a Insv site in the gene $\mathrm{vg}$ but not a mutated version. The shifted complexes can be competed away with cold probe and supershifted with Insv antibodies. $(B)$ Insv binds to endogenous sites from a variety of neural target genes. $(C)$ Schematics of actin-luciferase reporter constructs into which either wild-type or mutant Insv motifs or endogenous insv-bound genomic regions have been inserted. $(D)$ Insv-binding sites directly recruit Insv for transcriptional repression. Actin-luciferase reporters containing multimers of Insv-binding sites are repressed upon cotransfection of Insv plasmid relative to empty plasmid; no effects were observed with a similar reporter bearing point mutations in the Insv site. (E) Endogenous Insv-bound sites from a variety of neural target genes were similarly repressed. $(F)$ Insv variant proteins used for structure-function tests. $(G)$ Fusion of the VP16 activation domain to Insv or InsvC converts them into transcriptional activators; the latter demonstrates that DNA-binding activity localizes to the BEN domain.

(in a ribbon representation) bound per duplex. An alternate view of the complex with the BEN domain in an electrostatic surface representation is shown in Figure 3B. Overall, the BEN domain adopts a primarily $\alpha$-helical architecture (Fig. 3C,D). We detect a large number of intermolecular contacts in the complex, with each BEN domain interacting with both strands of the duplex. Helix $\alpha 5$ (residues 338-363) of the BEN domain is positioned within the major groove, while elements of a long loop (residues 297-319) between $\alpha 3$ and $\alpha 4$ are positioned in the minor groove of the duplex. We outline below intermolecular contacts between the green-colored Insv BEN domain and both strands of the 13-mer duplex. 
Table 1. Crystallographic statistics for Insv-BEN domain:DNA complexes

\begin{tabular}{|c|c|}
\hline \multicolumn{2}{|l|}{ Data collection } \\
\hline Wavelength & $0.9789 \AA$ \\
\hline Space group & P212121 \\
\hline \multicolumn{2}{|l|}{ Cell dimensions } \\
\hline$a, b, c$ & $43.4 \AA$ \\
\hline$\alpha, \beta, \gamma$ & $90^{\circ}, 90^{\circ}, 90^{\circ}$ \\
\hline Resolution $^{\mathrm{a}}$ & $50 \AA-1.58 \AA(1.61 \AA-1.58 \AA$ \\
\hline $\mathrm{R}_{\text {sym }}$ or $\mathrm{R}_{\text {merge }}{ }^{\mathrm{a}}$ & $0.084(0.712)$ \\
\hline$I / \sigma I$ & $59.6(4.0)$ \\
\hline Completeness $^{\mathrm{a}}$ & $99.9 \%(100 \%)$ \\
\hline Redundancy $^{\mathrm{a}}$ & $14.4(14.1)$ \\
\hline Unique reflections $^{\mathrm{a}}$ & 41,090 \\
\hline \multicolumn{2}{|l|}{ Refinement } \\
\hline Resolution & $39.06 \AA-1.58 \AA$ \\
\hline Number of reflections & 41,021 \\
\hline $\mathrm{R}_{\text {work }} / \mathrm{R}_{\text {free }}$ & $18.9 \% / 21.6 \%$ \\
\hline \multicolumn{2}{|l|}{ Number of atoms } \\
\hline Protein & 1812 \\
\hline DNA & 530 \\
\hline Water & 280 \\
\hline \multicolumn{2}{|l|}{ B factors } \\
\hline \multirow[t]{2}{*}{ Protein } & 25.2 \\
\hline & 19.5 \\
\hline Water & 30.6 \\
\hline \multicolumn{2}{|l|}{ RMS deviations } \\
\hline Bond lengths & $0.006 \AA$ \\
\hline Bond angles & $1.127^{\circ}$ \\
\hline
\end{tabular}

${ }^{a}$ Highest-resolution shell is shown in parentheses.

Intermolecular contacts of the BEN domain with the G1-T2-T3-C4-C5 segment of the 5' strand /colored light blue) are subdivided between base-specific (red box) and sugar-phosphate (black box) in the left panels of Figure 4. Key base-specific contacts include those between Asp351 and C4 and between Lys354 and G10' and G11' (Fig. 4, left panel, red box), while the side chains of basic residues Arg282, Arg291, and Lys348 as well as polar Ser278 form contacts with the sugar-phosphate backbone (Fig. 4, left panel, black box). Similarly, intermolecular contacts of the BEN domain with the $\mathrm{C}^{\prime}{ }^{\prime}-\mathrm{A} 6^{\prime}-\mathrm{A} 7^{\prime}-\mathrm{T} 8^{\prime}-\mathrm{T} 9^{\prime}$ segment of the $3^{\prime}$ strand (colored gold) are subdivided between base-specific (red box) and sugar-phosphate (black boxes) in the right panels of Figure 4. The side chain of Ser304 makes a base-specific contact with $\mathrm{A} 7{ }^{\prime}$, while the backbone amide of Ala306 makes a base-specific contact with T9 (Fig. 4, right panel, red box), with the side chains of basic residues Lys312, Lys315, Lys339, and Arg342 and polar residues Thr300, Thr346, and Asn353 as well as the backbone of Pro313 forming contacts with the sugarphosphate backbone (Fig. 4, right panel, black boxes).

All four thymine (T2, T3, T8', and T9') methyls form hydrophobic contacts in the major groove with BEN domain side chains, involving carbon-carbon distances between 3.7 and $4.0 \AA$. T2 is positioned opposite the $\mathrm{SCH}_{3}$ of Met355, T3 is positioned opposite $\mathrm{C} \beta$ of Asp351, T8' is positioned opposite methyl of Thr346, and T9' is positioned opposite $\mathrm{C} \beta$ of Ala350 and $\mathrm{C} \delta$ of Lys354. Overall, within the palindromic-like T2-T3-C4-C5-A6-A7T8-T9-G10-G11-A12 sequence, we observed base-specific intermolecular hydrogen-bonding contacts to the base pairs within the central (C4-C5-A6-A7).(T8'-T9'-G10'G11') segment and hydrophobic contacts to all four thymine methyls of the T2-T3 and T8'-T9' segments in the complex (Fig. 4). Importantly, the side chains of Asp351 and Lys354 on helix $\alpha 5$ form base-specific hydrogen-bond contacts in the major groove, while the side chain of Ser304 and the backbone amide of Ala306 form basespecific hydrogen-bond contacts in the minor groove in the complex (Fig. 4). We provide electron density maps for contacts of the BEN domain with the DNA backbone and specific nucleotide side chains in Supplemental Figure 3. Overall, these intermolecular contacts readily explain the specificity of the BEN domain of Insv for the TTCC AATTGGA-binding site.

\section{Functional validation of the Insv BEN:DNA complex}

We summarize the critical contacts of the BEN domain with the sugar-phosphate backbone (Fig. 5A) and with nucleotide side chains that determine binding specificity (Fig. 5B). We validated the crystal structure by assaying a series of alanine mutants of the BEN domain. With regard to BEN-DNA backbone interactions, gel shift studies revealed that $\mathrm{K} 315 \mathrm{~A}$ and $\mathrm{R} 342 \mathrm{~A}$ mutations resulted in complete loss in DNA binding, while partial loss in binding was observed for mutations of other interacting amino acids (Supplemental Fig. 4). Perhaps more significant was mutational analysis of base-specific amino acid contacts. As mentioned, Ser304 interacts with A7' , Asp351 recognizes $\mathrm{C} 4$, and Lys354 directly contacts two nucleotides, G10' and G11' (Fig. 5B). Gel shift studies showed that the S304A and D351A mutations resulted in partial loss in binding, while the K354A mutation nearly abolished binding (Fig. 5C) - the stronger effect of the latter mutation being consistent with the structural importance of this residue. The T347A mutant was not substantially compromised for DNA binding (Fig. 5C), highlighting the greater importance of the other contacting residues. Notably, several of these key DNA-contacting residues of the Insv BEN domain are conserved in other BEN domains (Fig. 6A).

We also tested reciprocal mutations of the target DNAbinding site. Consistent with the results of the BEN domain variants, mutation of $A 7^{\prime}$ to $G, T$, and $C_{\text {; }}$ mutation of $\mathrm{G} 11^{\prime}$ to $\mathrm{T}, \mathrm{A}$, or $\mathrm{C}$; and mutation of $\mathrm{G} 10^{\prime}$ to $\mathrm{T}$ or $\mathrm{C}$ all resulted in complete loss of binding by the Insv BEN domain. Interestingly, mutation of G10' to A was compatible with binding (Fig. 5D). Consistent with this, the TCYAATHRGAA consensus sequence from the ChIP-seq data indicated that position $10^{\prime}$ is occupied by $\mathrm{G}$ and $\mathrm{A}$ at similar frequencies (Fig. 1C).

Finally, we tested the effect of several BEN domain mutations on Insv function in vivo. We used the transcriptional assay in which mutant Insv proteins were challenged to repress the luciferase reporter bearing Insvbinding sites. Western blot analysis confirmed that these point mutant isoforms all accumulated stably (Fig. 5E), and immunofluorescence showed that all variants were appropriately localized to the nucleus (Fig. 5F; Supplemental 
Dai et al.
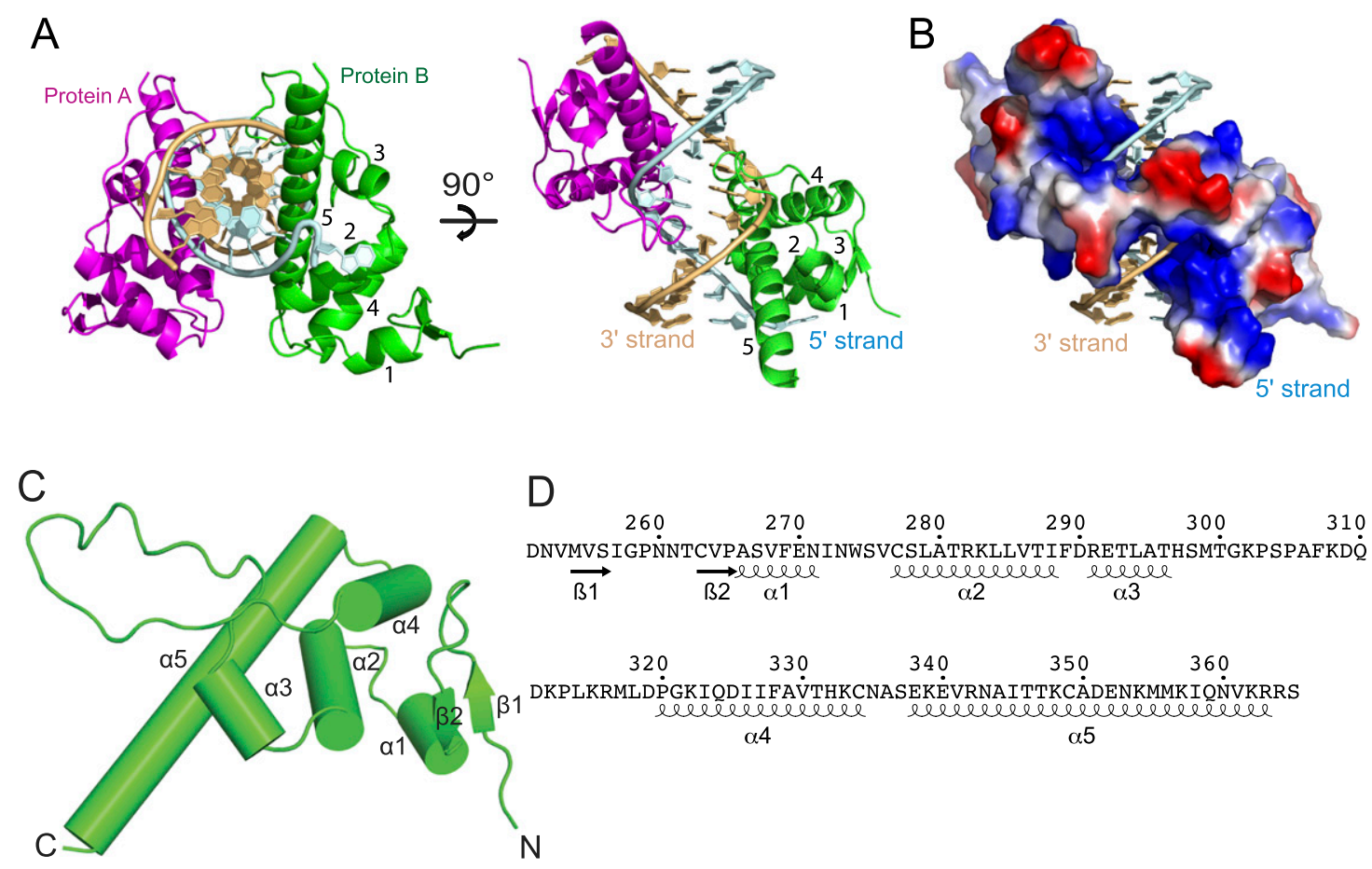

Figure 3. Overall structure of the Insv-BEN domain in the DNA-bound state. $(A)$ Two views of the structure of the complex containing two BEN domains (in ribbon representation) bound to a self-complementary 13-mer DNA duplex (strands colored in light blue and gold). The symmetry-related Ben domains are colored in green and magenta, with $\alpha$ helices numbered from 1 to $5 .(B)$ The same view of the complex as in the right panel of $A$, with protein in an electrostatic surface representation. $(C)$ Helical topology of the Insv Ben domain in complex with its DNA target site. Ribbon representation of the Insv BEN domain reveals a predominantly $\alpha$-helical structure. $(D)$ Positioning of $\alpha$ helices and $\beta$ strands within the Insv BEN domain.

Fig. 5). We observed that S304A was slightly compromised for repression but that D351A was strongly compromised and K354A was nearly inactive for transcriptional regulation; the D351A+K354A double mutant was completely inactive for transcriptional repression (Fig. 5G). These data correlate perfectly with the relative DNA-binding activities of these variants as assayed by electrophoresis mobility shift assays (EMSAs). Altogether, these data confirm our structural determination of intermolecular base-specific contacts in the Insv BEN domain complexed to DNA.

Conservation of DNA-binding activity in a mammalian neural BEN domain protein

Alignments of BEN domains from many species yield a loose consensus with a modest number of defining residues, none of which are completely conserved (Fig. 6A; Abhiman et al. 2008). Several amino acids that we demonstrated to be critical for DNA-binding activity of the Insv BEN domain are not shared by many instances of the motif; however, select proteins appear to maintain these particular residues. One such example is the vertebrate protein BEND5 (Fig. 6A), which shares key C-terminal tail residues such as Asp351 and Lys354. Vertebrate BEND5 orthologs exhibit very limited overall homology with Insv (8\% identity and $12 \%$ similarity) but share the layout of having only a potential N-terminal coiled-coil domain and a C-terminal BEN domain (Supplemental Fig. 6).
Perusal of in situ hybridization patterns collected by the Allen Brain Atlas database (http://www.brain-map.org) revealed that mouse BEND5 is similarly expressed in differentiating neurons in cortex (Supplemental Fig. 7). These patterns were recapitulated in BEND5::GFP-tagged bacterial artificial chromosome (BAC) transgenic mice produced by the GENSAT project (http://www.gensat.org/index.html) and confirm particularly strong expression in pyramidal projection neurons of layer V (Fig. 6B,C). Therefore, Insv and BEND5 define a family of neural-specific BEN-solo proteins.

We analyzed the regulatory capacity of human BEND5 on the Insv multimer reporter. Bearing in mind that S2 cells may not provide the appropriate context for hBEND5 function, we tested the hBEND5-BEN domain fused to VP16. We observed reporter activation that was specific to the wild-type Insv reporter, with no effect on the mutant reporter (Fig. 6D). Moreover, when expressed in HEK293T cells, full-length hBEND5 specifically inhibited the wildtype insv reporter (Fig. 6E), providing evidence for a conserved role of neural BEN-solo factors as transcriptional repressors. The activity of hBEND5 in these assays was modest compared with Insv, although it was possible that the Insv reporter tested did not contain its optimal binding site. We sought direct evidence of DNA binding by hBEND5 using gel shift analysis. We prepared a GSThBEND5-BEN domain fusion protein and observed that it was able to shift a radiolabeled probe containing the Insvbinding site from $v g$ but did not bind to a mutant probe 
Base-specific hydrogen-bonding contacts

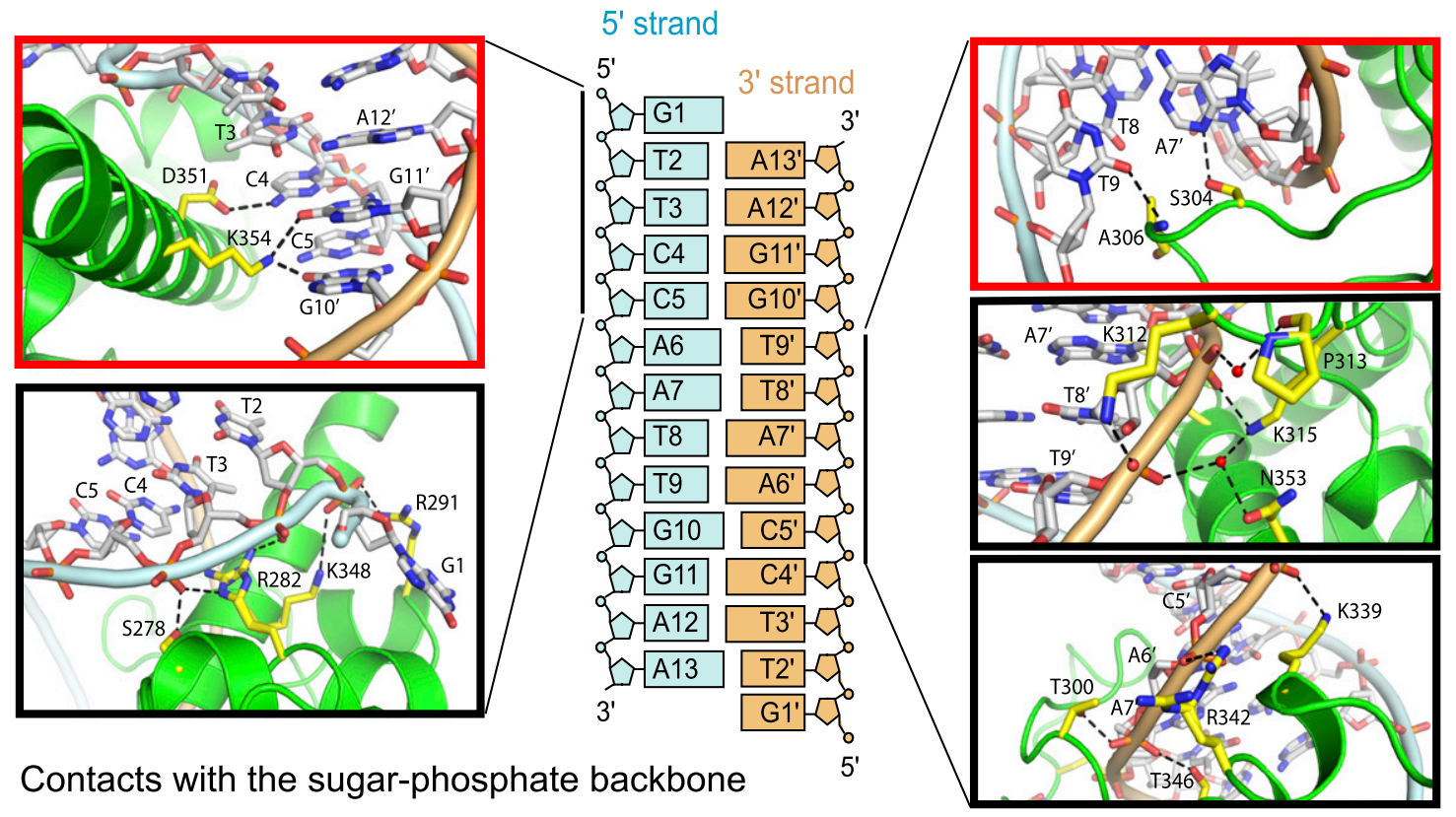

Figure 4. Amino acid contacts of the BEN domain with the DNA backbone and specific nucleotide side chains. Shown are details of intermolecular hydrogen-bonding contacts in the BEN-DNA complex. The sequence and numbering scheme are shown in the middle panel. Intermolecular hydrogen-bonding contacts involving the G1-T2-T3-C4-C5 segment on the 5' strand are shown in the panels on the left, while those involving contacts with the $\mathrm{C}^{\prime}-\mathrm{A} 6^{\prime}-\mathrm{A} 7^{\prime}-\mathrm{T} 8^{\prime}-\mathrm{T} 9^{\prime}$ of the $3^{\prime}$ strand are shown on the right. Base-specific hydrogenbonding contacts are shown in red boxes, while those involving contacts with the sugar-phosphate backbone are shown in black boxes.

(Fig. 6F). Therefore, DNA-binding activity of the BEN domain is conserved between these two otherwise quite disparate neural proteins from flies and human.

\section{Discussion}

Dual mechanisms for transcriptional regulation by Insensitive

We used molecular genetics and ChIP-seq analysis of the neural regulator Insv to delineate two strategies by which it associates with chromatin to mediate transcriptional repression. First, Insv functions as a corepressor for the transcription factor $\mathrm{Su}(\mathrm{H})$. In this mode of action, it is recruited to Notch target genes and can oppose the action of Notch signaling in activating these targets (Duan et al. 2011). Second, we found that the Insv BEN domain comprises a novel DNA-binding fold that directly recruits Insv homodimers to hundreds of target genes via a palindromic binding site. These dual activities permit Insv to act as a transcriptional repressor during neurogenesis by $\mathrm{Su}(\mathrm{H})$-dependent and $\mathrm{Su}(\mathrm{H})$-independent mechanisms, endowing it with functional versatility. As a corepressor tethered to DNA via $\mathrm{Su}(\mathrm{H})$, it tunes the appropriate transcriptional output of Notch target genes (Duan et al. 2011). It has long been appreciated that cell fate decisions regulated by the Notch pathway are exquisitely sensitive to the precise level of signaling (Heitzler and Simpson 1991). This appears to be manifest not only by the direct mechanism by which the Notch receptor transduces an extracellular signal into the nucleus, and is in fact obligately destroyed during this process (Lai 2004), but also by the fact that transcriptional activation by Notch is balanced by multiple mechanisms of transcriptional repression actively mediated by Notch transcription factors of the CSL family (Lai 2002). In Drosophila, Hairless serves as a major direct corepressor for $\mathrm{Su}(\mathrm{H})($ Morel et al. 2001; Barolo et al. 2002), and multiple stages of peripheral nervous system development require Hairless function, including during specification of the SOP and at multiple steps in the SOP lineage (Bang et al. 1991). However, misexpression of Insv is sufficient to fully rescue SOP specification in Hairless-null clones and can partially rescue their lineage divisions, attesting to its identity as a genuine $\mathrm{Su}(\mathrm{H})$ corepressor (Duan et al. 2011).

In this study, we show that Notch target genes comprise only a minor fraction of the totality of endogenous Insv target genes. Nevertheless, one of the most prominent categories of genes that contain Insv-occupied target sites are those involved in nervous system development. Indeed, we confirmed Insv binding and functional repression of a host of genes that regulate neural development, including vestigial, mir-263a, repo, fringe, grainyhead, hamlet, tramtrack, Synaptotagmin1, fne, and chinmo, among others (Figs. 1, 2). Direct transcriptional regulation by Insv provides a strategy for it to regulate neurogenesis independently of Notch signaling. In fact, the specific expression of Insv in SOPs (and within the lineage), its requirement for fully stable commitment to the SOP fate, and its capacity to promote ectopic peripheral sensory organs when misex- 
Dai et al.
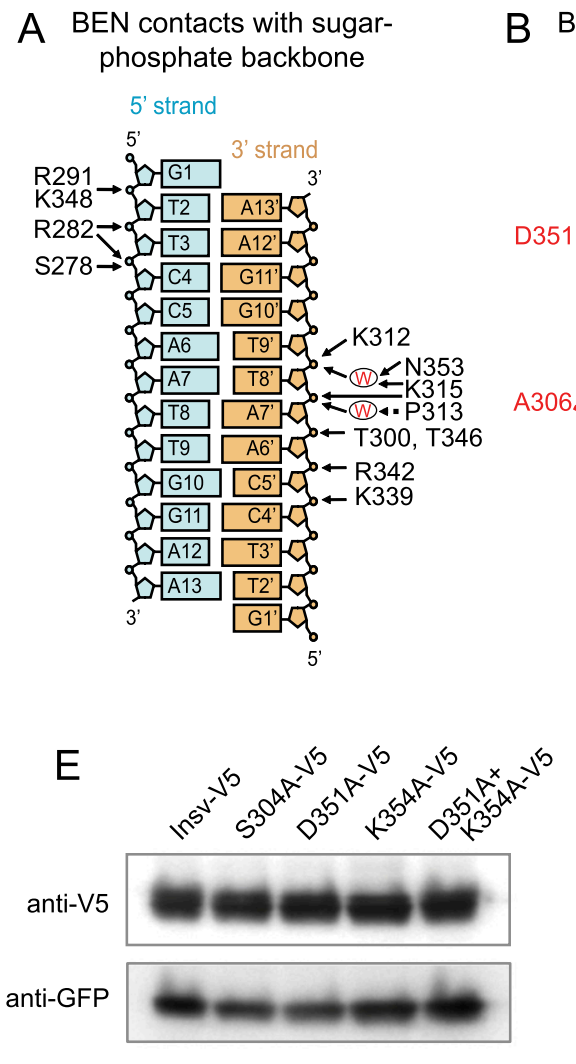
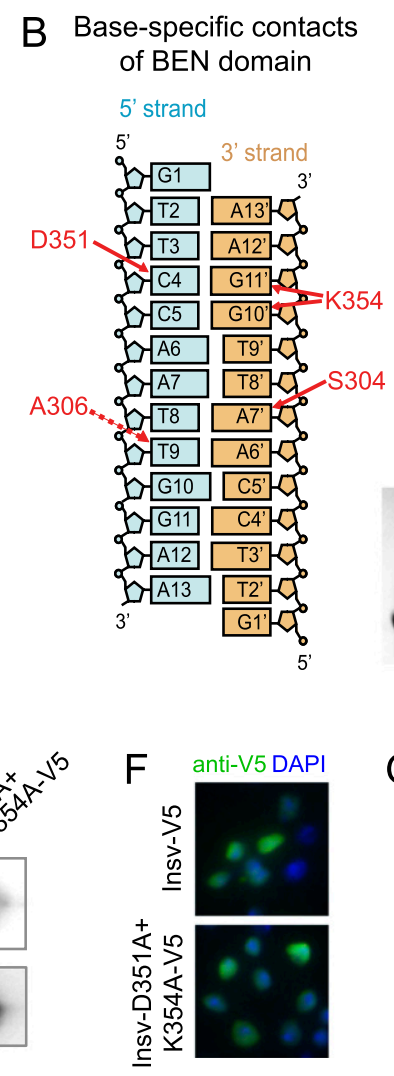

C Insv-BEN variants tested on wt probe

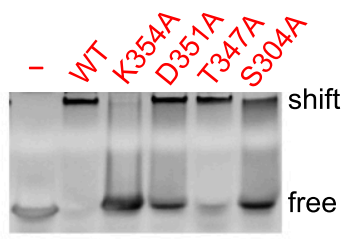

D WT Insv-BEN on variant probes

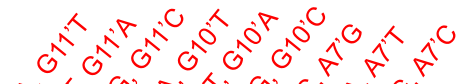

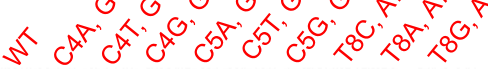

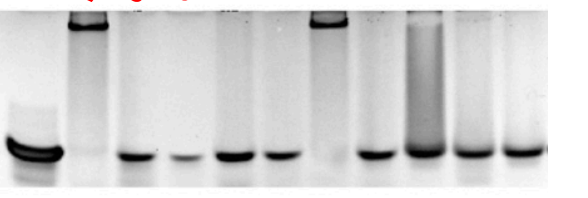

G

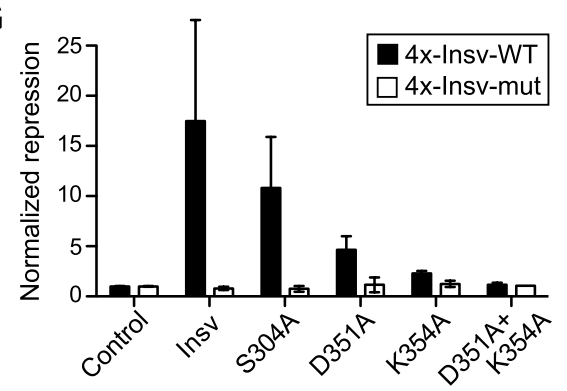

Figure 5. Functional assays confirm the structure of the BEN:DNA complex. (A) Summary of intermolecular hydrogen-bonding contacts to the sugar-phosphate backbone. (B) Summary of base-specific intermolecular hydrogen-bonding contacts. (C) Mutation of several BEN domain residues involved in base-specific recognition abrogates their functionality in gel shift assays visualized with ethidium bromide. $(D)$ Mutations of DNA base pairs involved in base-specific recognition by the BEN domain abrogate association with Insv-BEN in gel shift assays. (E) A panel of V5-tagged Insv proteins bearing alanine mutations of side chain-contacting residues in the BEN domain were transfected into S2 cells. Western analysis showed normal accumulation of all of the Insv variants. $(F)$ Immunostaining of Insv constructs in S2 cells showed that both wild type and the D351A+K354A double mutant exhibited nuclear localization (as did other mutant Insv proteins) (see Supplemental Fig. 5). (G) BEN domain mutants of Insv were cotransfected with wild-type and mutant insv transcriptional reporters. S304A had only mild effects, but D351A and K354A were strongly compromised for transcriptional repression. The D351A+K354A double mutant was completely inactive.

pressed (Duan et al. 2011) are all consistent with the viewpoint that Insv plays an active role in directing neurogenesis upstream of and/or in parallel to its role in inhibiting Notch signaling.

Our experimental data extensively support that Insv functions as a transcriptional repressor. Consistent with this, our catalog of validated Insv repression targets includes many genes that oppose neurogenesis (e.g., tramtrack), promote late temporal events in neural lineages (e.g., hamlet, grainyhead, and chinmo), or explicitly promote nonneural cell fates in the nervous system (e.g., the proglial factor repo). These observations support the notion that Insvmediated repression helps guide proper neural specification and cell fate determination within the neural lineage.

\section{Conservation of DNA-binding activity in other BEN domain proteins}

It is clear that the BEN consensus is modest at best, with no amino acids actually shared across all instances of this domain. Nevertheless, our structural studies draw particular attention to Insv residues that participate in direct contacts with the DNA backbone or that recognize specific DNA base side chains. This knowledge allows us to identify certain BEN domains as candidates for Insvlike binding specificity. For example, the BEN domain in the Drosophila Mod(mdg4)-PC (Abhiman et al. 2008) isoform retains few of the base-specific contacts of Insv, but two other Drosophila BEN-solo domain proteins bear striking homology with Insv across multiple residues that we defined as functionally critical for the DNA-binding activity of Insv (Fig. 6A, residues highlighted with asterisks). We predict that CG9883 and Bsg25A are good candidates for harboring sequence-specific DNA-binding activity.

Mammalian genomes encode many BEN domain proteins (Abhiman et al. 2008), but BEN domains per se have been little studied to date. We found that mammalian BEND5 is noticeably related to Insv across several functionally important DNA-binding residues, including at many positions within the $\mathrm{C}$-terminal tail region. Al- 


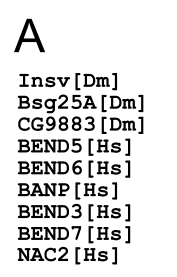

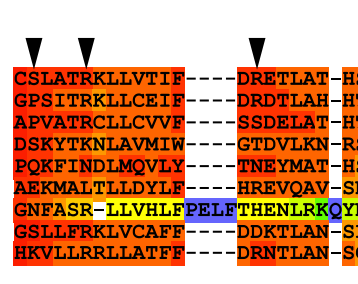

西
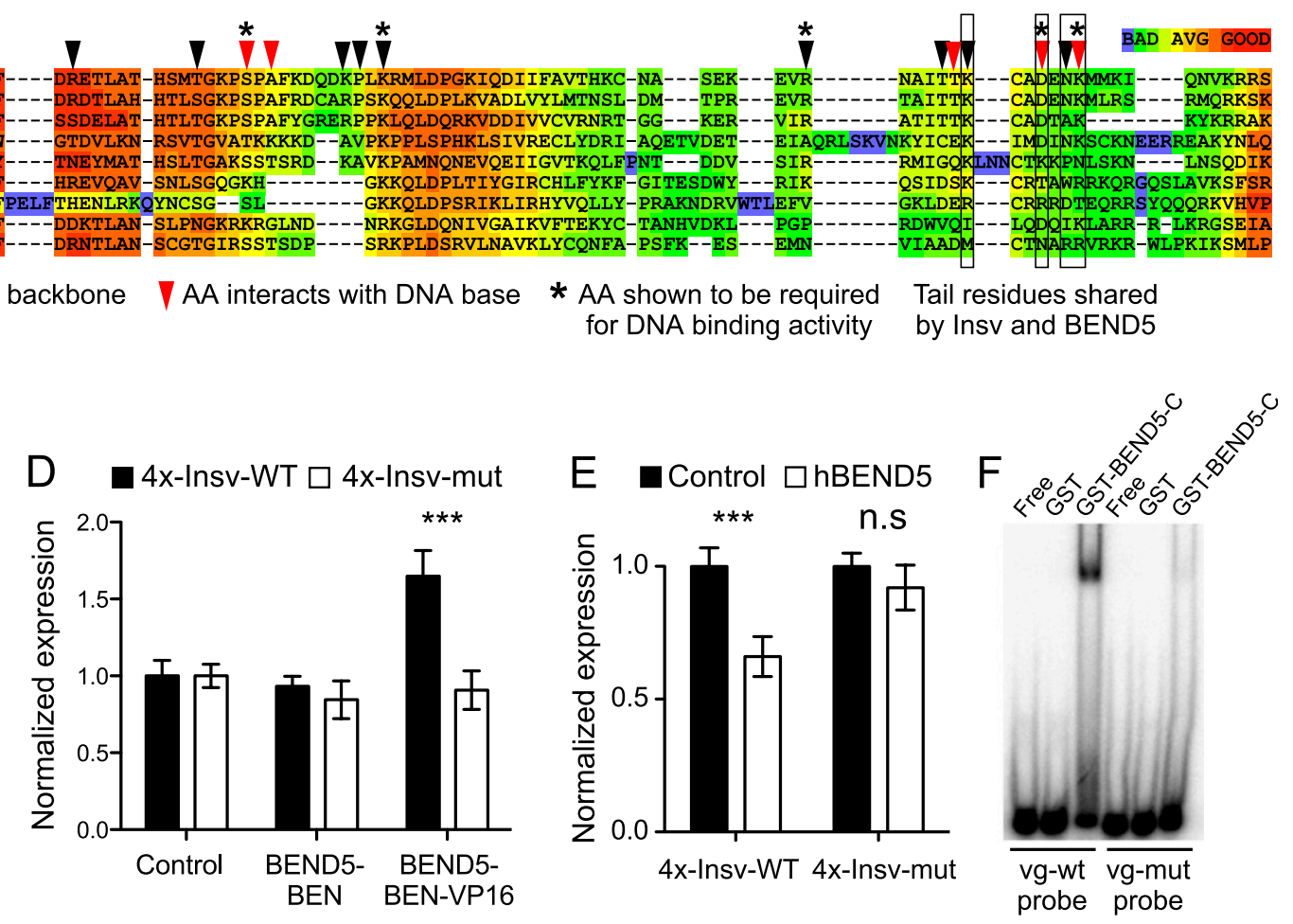

* AA shown to be required for DNA binding activity

Tail residues shared by Insv and BEND5

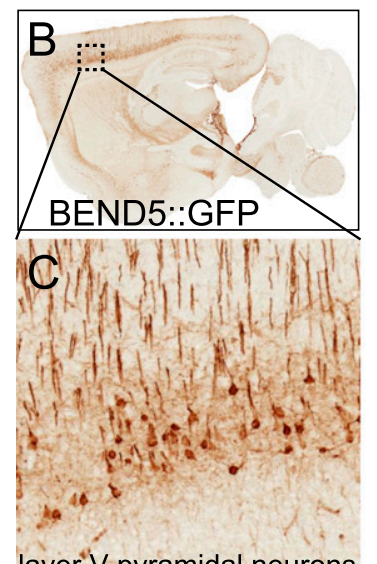

layer $V$ pyramidal neurons

Figure 6. Conservation of DNA-binding capacity in a mammalian neural BEN-solo protein. (A) Alignment of the Insv BEN domain (starting with the second $\alpha$ helix) (see Fig. 3) with BEN domains from other proteins reveals conservation of critical DNA-contacting residues in some Drosophila and mammalian proteins. (B) A BAC transgenic mouse bearing a knock-in of GFP into BEND5 exhibits specific expression in the cortex (data from the GENSAT project, http://www.gensat.org). The boxed region is enlarged in $C$ and highlights expression of BEND5 in layer V pyramidal neurons. (D) A VP16 fusion to the BEN domain of human BEND5 can activate the Insv reporter but does not affect the mutant reporter. (E) Full-length human BEND5 represses a wild-type but not mutant Insv reporter in HEK293T cells. $(F)$ Gel shift analysis using radiolabeled probes confirms specific binding of hBEND5 to a probe from the Drosophila $v g$ gene bearing an Insv site but not a $v g$ probe mutated at the Insv-binding site.

though it is possible that BEND5 may have a different optimal binding site, we were able to demonstrate specific binding of BEND5 to the Insv target site. Therefore, we can conclude that the BEN domain is a conserved DNA-binding domain. We identify other functional parallels between Insv and BEND5, including their specific expression in neurons and their capacity to function as transcriptional repressors. Given that Insv and BEND5 are fairly unrelated in primary sequence, these findings suggest that some of the other $>100$ BEN proteins are likely to be transcription factors. Curiously, some other BEN proteins, including the BTB/POZ-BEN factor NAC-1 and the quadruple BEN protein BEND3, have previously been linked to transcriptional repression (Korutla et al. 2009; Sathyan et al. 2011). Potentially, their BEN domains are involved in recruiting these factors to their appropriate chromatin targets.

Finally, not only are BEN domains widely distributed across metazoans, they are also encoded by several viral genomes (Abhiman et al. 2008). Our data suggest that BEN proteins may potentially prove to add to the catalog of cellular transcription factors that have been co-opted by viruses. Overall, our findings now provide molecular direction to the study of this large and enigmatic family of proteins.

\section{Materials and methods}

\section{Insv ChIP-seq and ChIP-qPCR}

We performed ChIP largely as previously described (Duan et al. 2011). In brief, 2.5- to 6.5-h and 6.5- to 12-h embryos from w[1118] (used as wild type) or insv[23B] homozygous mutants were aged, collected, dechorionated, and washed with water and $\mathrm{NaCl}$-Triton buffer. Embryos were homogenized and fixed in $1.8 \%$ formaldehyde in buffer A1 $160 \mathrm{mM} \mathrm{KCl}, 15 \mathrm{mM} \mathrm{NaCl}$, $4 \mathrm{mM} \mathrm{MgCl}_{2}, 15 \mathrm{mM}$ HEPES at $\mathrm{pH} 7.6,0.5 \%$ Triton X-100, 0.5 $\mathrm{mM}$ DTT, protease inhibitor cocktail [Roche]) for $15 \mathrm{~min}$ at room temperature. After three washes with buffer A1 and one wash with lysis buffer $(140 \mathrm{mM} \mathrm{NaCl}, 15 \mathrm{mM}$ HEPES at $\mathrm{pH} 7.6$, $1 \mathrm{mM}$ EDTA, $0.5 \mathrm{mM}$ EGTA, $0.1 \%$ sodium deoxycholate, $1 \%$ Triton, $0.5 \mathrm{mM}$ DTT), chromatin in lysis buffer containing $0.1 \%$ SDS was sheared by sonication on a Sonifier Bioruptor (Diagenode) to an average length of $0.5 \mathrm{~kb}$.

For each immunoprecipitation, $0.5 \mathrm{~mL}$ of supernatant containing sheared chromatin was incubated overnight with $40 \mu \mathrm{L}$ of Gammabind G agarose (GE Healthcare) coated with BSA to remove nonspecific background. The precleared chromatin was treated with either preabsorbed anti-Insv antibody or the preimmune serum, a reference sample, overnight. The precipitated immune complexes were washed and eluted, and cross-links were reversed overnight at $65^{\circ} \mathrm{C}$. After being treated with proteinase K, DNA was purified using QIAprep Spin Miniprep columns (Qiagen) and recovered in $50 \mu \mathrm{L}$ of elution buffer 
(Qiagen) containing RNase A. DNA libraries were made using the Illumina ChIP-seq library preparation kit and sequenced as individual lanes on the Illumina GAII.

Real-time PCR was performed on a Bio-Rad CFX96 machine using Power SYBR Green reagent (Applied Biosystems). Primers were designed with an online tool (Genescript). PCR was performed on $1 \mu \mathrm{L}$ of template DNA in triplicate samples, and immunoprecipitated DNA was compared against standard curves from serial dilutions of input DNA. The values are plotted as folds of enrichment normalized to the mock reference sample, and the standard deviation within the triplicate samples are indicated. Similar results were obtained in independent ChIP experiments.

\section{Processing of ChIP-seq data}

Reads were mapped to the Drosophila $\mathrm{dm} 3$ genome using Bowtie (Langmead et al. 2009) with parameters -v $2-\mathrm{m} 1$, (i.e., allowing two mismatches and only using uniquely mapping reads). Peaks were called using Quest (Valouev et al. 2008) with the following parameters: bandwidth, 30; region size, 300; ChIP enrichment, 10; ChIP to background enrichment, 2.5; and ChIP extension enrichment, 3. This resulted in a large set of peaks, which were ranked on the normalized enrichment fold $(\mathrm{EF})$ values for each. Peaks were annotated to the gene with the closest transcription start site using ENSEMBL transcript annotations.

\section{Motif finding}

We used two programs for de novo motif finding: Weeder (Pavesi et al. 2001) and MEME (Bailey and Elkan 1994). MEME was run on the top 500 peaks, extended 100 nucleotides (nt) in each direction from the top of the peak, to search for motifs 6-15 nt using default settings. Weeder was run on the same regions using the "medium" mode. To search for hits against the Insv site, the PWM Biostrings bioconductor package was used with a minimum score of $90 \%$. To compare the motif frequency in the genomic regions covered by the ChIP peaks to a background set, two strategies were used: (1) The peaks were shifted $1 \mathrm{~kb}$ in a random direction, and (2) regions the same size as the peaks, centered $250 \mathrm{bp}$ upstream of transcription start sites (but not overlapping the peaks), were sampled randomly. These approaches gave similar estimates of the background frequencies of the Insv site: Five percent of all shifted peak regions and $4 \%$ of all randomly sampled 200-bp regions upstream of transcription start sites contained the Insv site.

\section{Microarray analysis}

Six-hour-old to 8-h-old w[1118] (as wild-type control) and insv[23B] embryos were aged and collected for RNA extraction using Trizol reagent (Invitrogen). RNA samples from three biological replicates were tested by Agilent analyzer for quality control and labeled and hybridized to the Affymetrix GeneChIP Drosophila Genome 2.0 array in the Genomic Core Laboratory in Memorial Sloan-Kettering Cancer Center.

The microarray data were normalized using the GCRMA bioconductor package, and log enrichment values were computed using the limma package with $P$-values adjusted for multiple hypothesis using the false discovery rate (FDR) correction. For genes with multiple probe sets, the probe set with the lowest adjusted $P$-value was selected. To detect broad patterns of changes in gene expression, empirical cumulative distributions were computed using the R ecdf function on mutually exclusive gene sets, and $P$-values were computed by the Wilcoxson rank sum nonparametric test. Here, only ChIP-seq peaks that were within $10 \mathrm{~kb}$ of the closest transcription start site were considered.

Enrichment of GO annotations (Ashburner et al. 2000) and tissue-enriched gene expression profiles (Chintapalli et al. 2007) were computed with Fisher's exact test using the Bonferroni correction for multiple hypothesis testing. FlyAtlas gene classifications were based on tissue enrichment scores; genes with enrichment scores of at least 2 were considered to be enriched in a given tissue.

\section{Luciferase reporter assays}

An Insv-V5 fusion construct was generated by inserting an insv ORF sequence with a mutated stop codon to the NotI and XhoI sites of the pAcV5/HisA vector (Invitrogen). To mutate the corresponding bases in the residues, Ser304, Asp351, and Lys354 primer oligos encoding mutant residues were designed, and site mutagenesis was performed according to Stratagene site mutagenesis protocol. Insv-N, Insv-C, and BEND5C fragments were created by PCR amplification from Insv and BEND5 cDNAs with primers containing EcoRI and XhoI sites (sequences listed in Supplemental Table 4) and cloned into the EcoRI and XhoI sites of pAcV5/HisA (Invitrogen). These plasmids were digested with $\mathrm{XhoI}$ and $\mathrm{XbaI}$ sites and ligated with a DNA fragment with the same sites encoding VP16 activation domain, yielding pAc-InsvVP16, pAc-InsvN-VP16, pAc-InsvC-VP16, and pAc-BEND5CVP16 constructs. The full-length BEND5 ORF was obtained from pOTB7-BEND5 plasmid (American Type Culture Collection) and cloned to the pCAG-IRES-GFP vector. The Insv reporter used a 30nt region of the vestigial gene centered on an Insv motif or a mutant version carrying four nucleotide changes in the Insv site. Oligos containing two copies of this sequence were ligated through a designed Xhol site to obtain four copies of Insv motif (4xwt and 4xmut). These ligated fragments were then cloned into pAc-2T-Luc (Ryu and Arnosti 2003) using the AscI site to obtain pAc-4xwtLuc and pAc-4xmut-Luc for S2 cell transfection or into pcDNA3Luc (Addgene) using the HindIII site for HEK293T cell transfection.

\section{qRT-PCR}

Wild-type or insv mutant embryos $(6-8 \mathrm{~h})$ were collected and aged before RNA extraction. Total RNA was isolated by use of TRIzol reagent (Invitrogen) and further cleaned with Turbo DNase (Ambion). Four micrograms of total RNA was reverse-transcribed with SuperScript III reverse transcriptase (Invitrogen) using random primer mix (New England Biolabs). To ensure the absence of genomic DNA, qPCR was performed on a mock-reversetranscribed RNA sample. Primer sequences are listed in Supplemental Table 4.

\section{GST fusion protein purification and EMSAs}

Insv, InsvN, InsvC, and BEND5C fragments were PCR-amplified and cloned into EcoRI and XhoI sites in pGEX5x-2 (Insv, InsvN, and InsvC) or pGEX5x-1 (BEND5C) using primers as listed in Supplemental Table 1. GST and GST fusion proteins were expressed in Escherichia coli BL21 cells by induction with IPTG for $5 \mathrm{~h}$ at room temperature and purified on glutathione sepharose beads (Sigma). Purified GST fusion proteins were eluted in elution buffer containing $20 \mathrm{mM}$ glutathione (Sigma) in $50 \mathrm{mM}$ Tris- $\mathrm{HCl}$ ( $\mathrm{pH} 8.0$ ) and concentrated by using Amicon 0.5-mL centrifugal filters (Millipore).

To obtain dsDNA probes, sense oligos were labeled with 32P $\gamma$-ATP using T4 polynucleotide kinase (New England Biolabs), purified with G-25 columns (GE Healthcare), and annealed with complementary antisense oligos. One microgram of GST, GST- 
InsvC, or GST-BEND5C was mixed with 0.5-ng DNA probes and incubated for $30 \mathrm{~min}$ at room temperature. In the antibody supershift sample, $2 \mu \mathrm{L}$ of Insv antibody was added to the mixture and incubated for another $30 \mathrm{~min}$. These samples were then loaded to $4 \%$ native polyacrylamide gel. After $2-3 \mathrm{~h}$ running in a cold room, gels were vacuum-dried at $80^{\circ} \mathrm{C}$ and exposed to a PhosphorImager (Fuji Film). All oligo sequences are included in Supplemental Table 4.

Some nonradioactive EMSAs were performed on polyacrylamide gel (PAGE) and visualized using ethidium bromide. Here, the 13-mer self-complementary DNA duplex containing a central palindromic TCCAATTGGA-binding site or mutants in specific positions of the binding sites were used to characterize DNA binding of the Insv-BEN(251-365) domain or its mutants. Binding reactions contained $1.2 \mathrm{nmol}$ of dsDNA and $12 \mathrm{nmol}$ of protein dissolved in $13 \mu \mathrm{L}$ of binding buffer (20 mM HEPES, 150 $\mathrm{mM} \mathrm{NaCl}, 5 \mathrm{mM}$ DTT, $20 \mathrm{mM} \mathrm{MgCl}_{2}$ at $\mathrm{pH}$ 6.8). Binding reaction mixtures were electrophoresed in $10 \%$ PAGE in buffer (50 mM Tris-acetate, $50 \mathrm{mM}$ K-acetate, $5 \mathrm{mM} \mathrm{Mg}$-acetate at $\mathrm{pH}$ $8.0)$ at $4^{\circ} \mathrm{C}$. DNA was visualized by ethidium bromide staining.

\section{Cell culture and transient transfection}

Drosophila S2- $\mathrm{R}^{+}$cells were grown in Schneider Drosophila medium containing $10 \%$ fetal calf serum, $1 \times$ Glutamax (Gibco), $0.05 \mathrm{mg} / \mathrm{mL}$ gentamycin, $50 \mathrm{IU} / \mathrm{mL}$ penicillin, and $50 \mu \mathrm{g} / \mathrm{mL}$ streptomycin. Cells were transfected in a 96-well plate using the Effectene transfection kit (Invitrogen). In each well, $0.1 \times 10^{6}$ cells in $100 \mu \mathrm{L}$ of medium were transfected with $50 \mathrm{ng}$ of pAc4xwt-Luc or pAc-4xmut-Luc reporter together with $10 \mathrm{ng}$ of pAcrenilla-Luc (Saj et al. 2010), cotransfected with 200 ng of pAcV5/ His vector as control, or cotransfected with pAc-Insv, pAc-InsvN, pAc-InsvC, pAc-Insv-VP16, pAc-InsvN-VP16, pAc-InsvC-VP16, pAc-BEND5C, pAC-BEND5C-VP16. Cells were harvested after $72 \mathrm{~h}$ of incubation. Luciferase activity was measured in living cells using the Dual Luciferase assay system (Promega), and expression was calculated as the ratio between the firefly and Renilla luciferase activities.

HEK-293T cells were cultured in Dulbecco's modified Eagle medium (Gibco) containing $10 \%$ fetal calf serum, $50 \mathrm{IU} / \mathrm{mL}$ penicillin, and $50 \mu \mathrm{g} / \mathrm{mL}$ streptomycin. Transfections were performed by using Lipofectamine 2000 reagent (Invitrogen) following the manufacturer's instructions. For each well, $100 \mu \mathrm{L}$ of cells with the density of $2 \times 10^{5}$ per milliliter were seeded $24 \mathrm{~h}$ before transfection. Fifty nanograms of $4 x w t-L u c$ or pAc-4xmutLuc reporter together with $10 \mathrm{ng}$ of pCMV-renilla-Luc were cotransfected with empty vector or pCAG-BEND5. Luciferase activity was measured $24 \mathrm{~h}$ after transfection.

\section{Protein purification and crystallization conditions}

The sequence corresponding to 251-365 of Insv was inserted into a modified pRSFDuet-1 vector (Novagen) in which Insv-BEN domains were separated from a preceding His6-SUMO tag by a ubiquitin-like protease (ULP1) cleavage site. Proteins were overexpressed in E. coli BL21(DE3) and purified through a NiNTA affinity column. The His6-SUMO tag was removed by ULP1 cleavage during dialysis against buffer containing $25 \mathrm{mM}$ Tris ( $\mathrm{pH} 8.0$ ), $0.5 \mathrm{M} \mathrm{NaCl}$, and $5 \mathrm{mM} \beta$-mercaptoethanol. After dialysis, the protein sample was further fractionated through a second Ni-NTA affinity column and a SP column (GE Healthcare) followed by gel filtration on a 16/60 G75 Superdex column (GE Healthcare). The final sample for crystallization was concentrated to $\sim 20 \mathrm{mg} / \mathrm{mL}$ protein in buffer containing $20 \mathrm{mM}$ HEPES, $150 \mathrm{mM} \mathrm{NaCl}, 5 \mathrm{mM}$ DTT, and $20 \mathrm{mM} \mathrm{MgCl}$ (pH 6.8). Approximately $2 \mathrm{mg}$ was usually produced from each liter of cell culture.
To generate the BEN-DNA complex, a 13-mer self-complementary DNA duplex containing a central palindromic TCCAATTGGAbinding site was incubated with Insv-BEN(251-365) in a 1:1.21.5 molar ratio on ice for $1 \mathrm{~h}$. The initial crystallization condition (0.1 M MES at pH 6.5, PEG400 30\%) for Insv-BEN(251-365)DNA complex was identified using PEG suite screens (Qiagen). The crystals were subsequently reproduced by hanging-drop vapor diffusion method at $20^{\circ} \mathrm{C}$ from drops mixed from $1 \mu \mathrm{L}$ of Insv-DNA solution and $1 \mu \mathrm{L}$ of precipitant solution $(0.1 \mathrm{M}$ MES at $\mathrm{pH}$ 6.5, PEG400 26\%). Crystals were directly flash frozen in liquid nitrogen for data collection.

\section{Structure determination}

X-ray diffraction data sets for the Insv-BEN(251-365)-DNA complex were collected at selenium peak wavelength on the X29A beamline at the National Synchrotron Light Source (NSLS). The diffraction data were indexed, integrated, and scaled using the HKL 2000 program (HKL Research). The structure (space group: $\mathrm{P} 2{ }_{1} 2_{1} 2_{1}$ ) was determined using single-wavelength anomalous dispersion $(\mathrm{SAD})$ technique using anomalous signal from four selenium atoms with the HKL2MAP program (Pape and Schneider 2004) and PHENIX suite (Adams et al. 2002). There was one molecule of the Insv-BEN(251-365)-DNA complex in the asymmetric unit (ASU). Each DNA duplex molecule bound to two protein molecules. Further modeling of the Insv-BEN(251-365)DNA complex was carried out using COOT (Emsley and Cowtan 2004) and was then subjected to refinement using PHENIX. The statistics for data collection and structural refinement of the Insv-BEN(251-365)-DNA complex are summarized in Table 1. The coordinates of the Insv-BEN(251-365)-DNA complex have been deposited in the Research Collaboratory for Structural Bioinformatics (RCSB) Protein Data Bank with accession number 4IX7.

\section{Acknowledgments}

We thank Alexander Serganov and Jiamu Du for guidance and assistance in BEN construct design, and the staff of beamline X-29 at the Brookhaven National Laboratory for assistance with data collection. We are especially grateful to the Allen Brain Atlas and GENSAT project for making their expression data publicly available. D.J.P. was supported by funds from the Abby Rockefeller Mauze Trust and the Maloris Foundation. Q.D. and J.O.W. were supported by fellowships from the Swedish Research Council. Work in E.C.L.'s group was supported by the Burroughs Wellcome Fund and the National Institute of Neurological Disorders and Stroke of the National Institutes of Health under award number 1R01-NS074037. The content is solely the responsibility of the authors and does not necessarily represent the official views of these funding agencies. Q.D. was involved in project design, generated the ChIP-seq and microarray data, and performed all of the target gene validation and reporter construct assays. J.O.W. performed all bioinformatic analysis. A.S. was involved in BEN construct design, while A.R. generated the complex, crystallized it, and solved the structure under the supervision of D.J.P. E.C.L. was involved in the experimental design and interpretation and wrote the manuscript with input from the coauthors.

\section{Note added in proof}

Schedl and colleagues (Aoki et al. 2012) recently reported that CG9883 and Bsg25A are components of a DNA-binding complex. The ChIP-seq and microarray data were deposited at the Gene Expression Omnibus under accession GSE44178. 
Dai et al.

\section{References}

Abhiman S, Iyer LM, Aravind L. 2008. BEN: A novel domain in chromatin factors and DNA viral proteins. Bioinformatics 24: 458-461.

Adams PD, Grosse-Kunstleve RW, Hung LW, Ioerger TR, McCoy AJ, Moriarty NW, Read RJ, Sacchettini JC, Sauter NK, Terwilliger TC. 2002. PHENIX: Building new software for automated crystallographic structure determination. Acta Crystallogr D Biol Crystallogr 58: 1948-1954.

Aoki T, Sarkeshik A, Yates J, Schedl P. 2012. Elba, a novel developmentally regulated chromatin boundary factor is a hetero-tripartite DNA binding complex. eLife 1: e00171.

Ashburner M, Ball CA, Blake JA, Botstein D, Butler H, Cherry JM, Davis AP, Dolinski K, Dwight SS, Eppig JT, et al. 2000. Gene ontology: Tool for the unification of biology. The Gene Ontology Consortium. Nat Genet 25: 25-29.

Bailey TL, Elkan C. 1994. Fitting a mixture model by expectation maximization to discover motifs in biopolymers. Proc Int Conf Intell Syst Mol Biol 2: 28-36.

Bang AG, Hartenstein V, Posakony JW. 1991. Hairless is required for the development of adult sensory organ precursor cells in Drosophila. Development 111: 89-104.

Barolo S, Stone T, Bang AG, Posakony JW. 2002. Default repression and Notch signaling: Hairless acts as an adaptor to recruit the corepressors Groucho and dCtBP to Suppressor of Hairless. Genes Dev 16: 1964-1976.

Boch J, Scholze H, Schornack S, Landgraf A, Hahn S, Kay S, Lahaye T, Nickstadt A, Bonas U. 2009. Breaking the code of DNA binding specificity of TAL-type III effectors. Science 326: $1509-1512$.

Chintapalli VR, Wang J, Dow JA. 2007. Using FlyAtlas to identify better Drosophila melanogaster models of human disease. Nat Genet 39: 715-720.

Dolfini D, Gatta R, Mantovani R. 2012. NF-Y and the transcriptional activation of CCAAT promoters. Crit Rev Biochem Mol Biol 47: 29-49.

Duan H, Dai Q, Kavaler J, Bejarano F, Medranda G, Nègre N, Lai EC. 2011. Insensitive is a novel corepressor of Suppressor of Hairless and regulates Notch-mediated cell fate decisions in the peripheral nervous system. EMBO J 30: 3120-3133.

Emsley P, Cowtan K. 2004. Coot: Model-building tools for molecular graphics. Acta Crystallogr D Biol Crystallogr 60: 2126-2132.

Heitzler P, Simpson P. 1991. The choice of cell fate in the epidermis of Drosophila. Cell 64: 1083-1092.

Korutla L, Wang P, Jackson TG, Mackler SA. 2009. NAC1, a $\mathrm{POZ/BTB}$ protein that functions as a corepressor. Neurochem Int 54: 245-252.

Lai EC. 2002. Keeping a good pathway down: Transcriptional repression of Notch pathway target genes by CSL proteins. EMBO Rep 3: 840-845.

Lai EC. 2004. Notch signaling: Control of cell communication and cell fate. Development 131: 965-973.

Lai EC, Orgogozo V. 2004. A hidden program in Drosophila peripheral neurogenesis revealed: Fundamental principles underlying sensory organ diversity. Dev Biol 269: 1-17.

Langmead B, Trapnell C, Pop M, Salzberg SL. 2009. Ultrafast and memory-efficient alignment of short DNA sequences to the human genome. Genome Biol 10: R25.

Lindblad-Toh K, Garber M, Zuk O, Lin MF, Parker BJ, Washietl S, Kheradpour P, Ernst J, Jordan G, Mauceli E, et al. 2011. A high-resolution map of human evolutionary constraint using 29 mammals. Nature 478: 476-482.

Lohse MB, Zordan RE, Cain CW, Johnson AD. 2010. Distinct class of DNA-binding domains is exemplified by a master regulator of phenotypic switching in Candida albicans. Proc Natl Acad Sci 107: 14105-14110.

Morel V, Lecourtois M, Massiani O, Maier D, Preiss A, Schweisguth F. 2001. Transcriptional repression by Suppressor of Hairless involves the binding of a Hairless-dCtBP complex in Drosophila. Curr Biol 11: 789-792.

Moscou MJ, Bogdanove AJ. 2009. A simple cipher governs DNA recognition by TAL effectors. Science 326: 1501.

Nellesen DT, Lai EC, Posakony JW. 1999. Discrete enhancer elements mediate selective responsiveness of Enhancer of split complex genes to common transcriptional activators. Dev Biol 213: 33-53.

Neph S, Vierstra J, Stergachis AB, Reynolds AP, Haugen E, Vernot B, Thurman RE, John S, Sandstrom R, Johnson AK, et al. 2012. An expansive human regulatory lexicon encoded in transcription factor footprints. Nature 489: 83-90.

Pape T, Schneider TR. 2004. HKL2MAP: A graphical user interface for phasing with SHELX programs. I Appl Crystal$\log$ 37: 843-844.

Pavesi G, Mauri G, Pesole G. 2001. An algorithm for finding signals of unknown length in DNA sequences. Bioinformatics 17: S207-S214.

Reeves N, Posakony JW. 2005. Genetic programs activated by proneural proteins in the developing Drosophila PNS. Dev Cell 8: 413-425.

Roy S, Ernst J, Kharchenko PV, Kheradpour P, Negre N, Eaton ML, Landolin JM, Bristow CA, Ma L, Lin MF, et al. 2010. Identification of functional elements and regulatory circuits by Drosophila modENCODE. Science 330: 1787-1797.

Ryu JR, Arnosti DN. 2003. Functional similarity of Knirps CtBPdependent and CtBP-independent transcriptional repressor activities. Nucleic Acids Res 31: 4654-4662.

Saj A, Arziman Z, Stempfle D, van Belle W, Sauder U, Horn T, Durrenberger M, Paro R, Boutros M, Merdes G. 2010. A combined ex vivo and in vivo RNAi screen for notch regulators in Drosophila reveals an extensive notch interaction network. Dev Cell 18: 862-876.

Sathyan KM, Shen Z, Tripathi V, Prasanth KV, Prasanth SG. 2011. A BEN-domain-containing protein associates with heterochromatin and represses transcription. J Cell Sci 124: 3149-3163.

Stark A, Lin MF, Kheradpour P, Pedersen JS, Parts L, Carlson JW, Crosby MA, Rasmussen MD, Roy S, Deoras AN, et al. 2007. Discovery of functional elements in 12 Drosophila genomes using evolutionary signatures. Nature 450: 219-232.

Valouev A, Johnson DS, Sundquist A, Medina C, Anton E, Batzoglou S, Myers RM, Sidow A. 2008. Genome-wide analysis of transcription factor binding sites based on ChIP-Seq data. Nat Methods 5: 829-834.

Wiedenheft B, Zhou K, Jinek M, Coyle SM, Ma W, Doudna JA. 2009. Structural basis for DNase activity of a conserved protein implicated in CRISPR-mediated genome defense. Structure 17: 904-912.

Yusuf D, Butland SL, Swanson MI, Bolotin E, Ticoll A, Cheung WA, Zhang XY, Dickman CT, Fulton DL, Lim JS, et al. 2012. The transcription factor encyclopedia. Genome Biol 13: R24. 


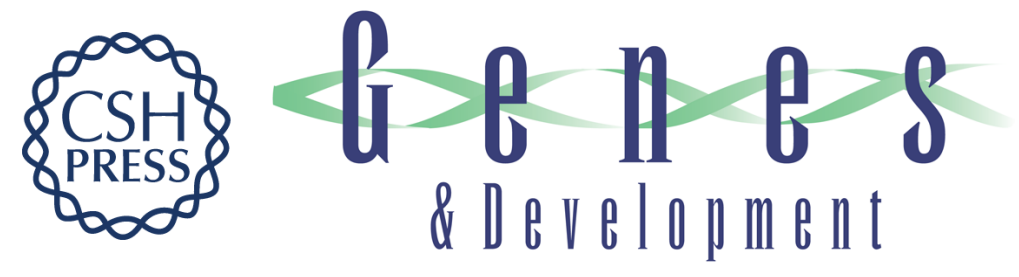

\section{The BEN domain is a novel sequence-specific DNA-binding domain conserved in neural transcriptional repressors}

Qi Dai, Aiming Ren, Jakub O. Westholm, et al.

Genes Dev. 2013, 27: originally published online March 6, 2013

Access the most recent version at doi:10.1101/gad.213314.113

\section{Supplemental http://genesdev.cshlp.org/content/suppl/2013/02/27/gad.213314.113.DC1 Material}

References This article cites 35 articles, 9 of which can be accessed free at: http://genesdev.cshlp.org/content/27/6/602.full.html\#ref-list-1

\section{License}

Email Alerting

Receive free email alerts when new articles cite this article - sign up in the box at the top Service

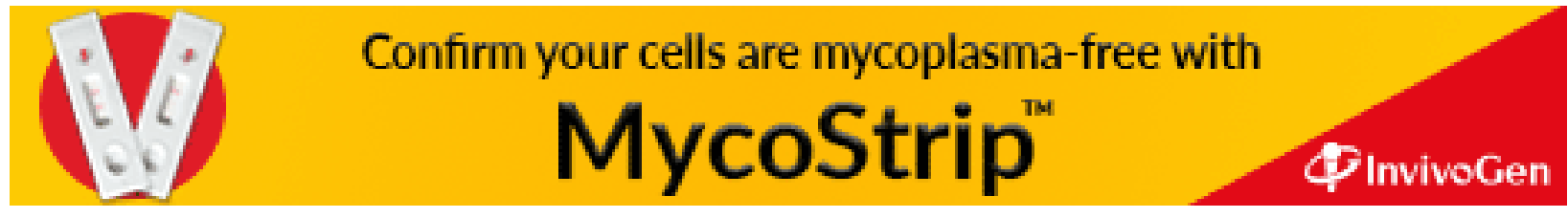

